\title{
Prise en compte d'un module de réaction corrigé dans la modélisation numérique d'un pieu isolé chargé latéralement à proximité d'une pente
}

\author{
Fidèle Massamba ${ }^{1}$, Daniel Levacher ${ }^{2}$, Salim Mezazigh ${ }^{3}$ \\ ${ }^{1}$ Docteur, ${ }^{2}$ Professeur, ${ }^{3}$ Maître de Conférences ; Université de Caen ; \\ M2C-GRGC-UMR 6143 CNRS : 24, rue des Tilleuls, 14000 CAEN \\ fidele.massamba@unicaen.fr
}

\begin{abstract}
Résumé : L'apport de résultats expérimentaux issus d'essais sur modèles centrifugés a permis d'envisager une simulation numérique d'un pieu isolé implanté à proximité d'un talus et soumis à un chargement monotone. Pour ce faire, un code de calcul en éléments finis a été utilisé pour modéliser le comportement d'un tel pieu installé dans un sable sec en bordure de différentes pentes. La prise en compte d'un modèle de réaction-corrigé de par l'introduction de coefficients réducteurs $R_{P}$ est proposée à partir de la relation de Christoulas et d'une confrontation expérimentale et numérique. D'une part la relation de Christoulas a été modifiée et d'autre part des coefficients de réduction pour deux pentes usuelles de talus ont été déduits. Ainsi la présence du talus qui affecte l'évolution du module de réaction en fonction de la profondeur et de la distance à la pente est considérée pour aborder l'étude du comportement d'un pieu implanté en bordure d'un talus.
\end{abstract}

\begin{abstract}
Experimental results obtained using centrifuge allows one to do numerical modelling of a pile in the proximity of an embankment, subjected to monotonic loading. To accomplish such, a finite element code using ANSYS 8.0 was utilized to model the behaviour of a pile installed in dry sand in the vicinity of a slope with varying distance. Taking into account of the reaction of the pile, a correction factor, $\mathrm{Rp}$, was introduced based on the relationship proposed by Christoulas and the results from numerical and experimental simulation. On one hand, the relation of Christoulas was modified and on the other coefficient of reduction of two different embankment slopes was derived. Thus, it has been shown that the proximity of slope affects the evolution of reaction module as a function of depth as well distance from the slope.
\end{abstract}

Mots clés : Interaction pieu-sol, chargement latéral, module de réaction, éléments finis, talus, modèles centrifugés.

Keywords : pile-soil interaction, lateral load, reaction modulus, finite elements method, slope, centrifuge modeling.

\section{Introduction}


Le calcul des déformations d'un pieu isolé chargé latéralement implanté dans un massif de sable à proximité d'une pente, impose souvent le recours à une méthode numérique. La difficulté réside dans la détermination du module de réaction du sol $E_{S}$. Le module de réaction caractérise le comportement élastique du sol. Une première confrontation numérique-expérimentale a permis dans le cas d'un pieu fiché dans un sol horizontal, de définir et caractériser l'évolution du module de réaction. Deux simulations numériques ont été menées en considérant un module de réaction constant avec la profondeur $\mathrm{z}$ puis un module évoluant avec $\mathrm{z}$. Ceci a débouché sur la proposition d'une modification de la relation de Christoulas. S'ensuit la modélisation du système pieu-sol en présence d'un talus. L'influence de la présence du talus a été prise en compte par la proposition de coefficients réducteurs $R_{P}$ correspondant aux pentes $3 / 2$ et $2 / 1$. La combinaison de la relation de Christoulas modifiée associée aux coefficients de réduction $R_{P}$ a permis d'optimiser les réponses (déplacements et moments fléchissants) d'un pieu isolé chargé latéralement et de confronter les solutions numériques ainsi obtenues pour les talus aux réponses expérimentales.

\section{Description du modèle numérique}

La géométrie du massif de sable est en relation avec la taille des conteneurs utilisés lors des essais en centrifugeuse.

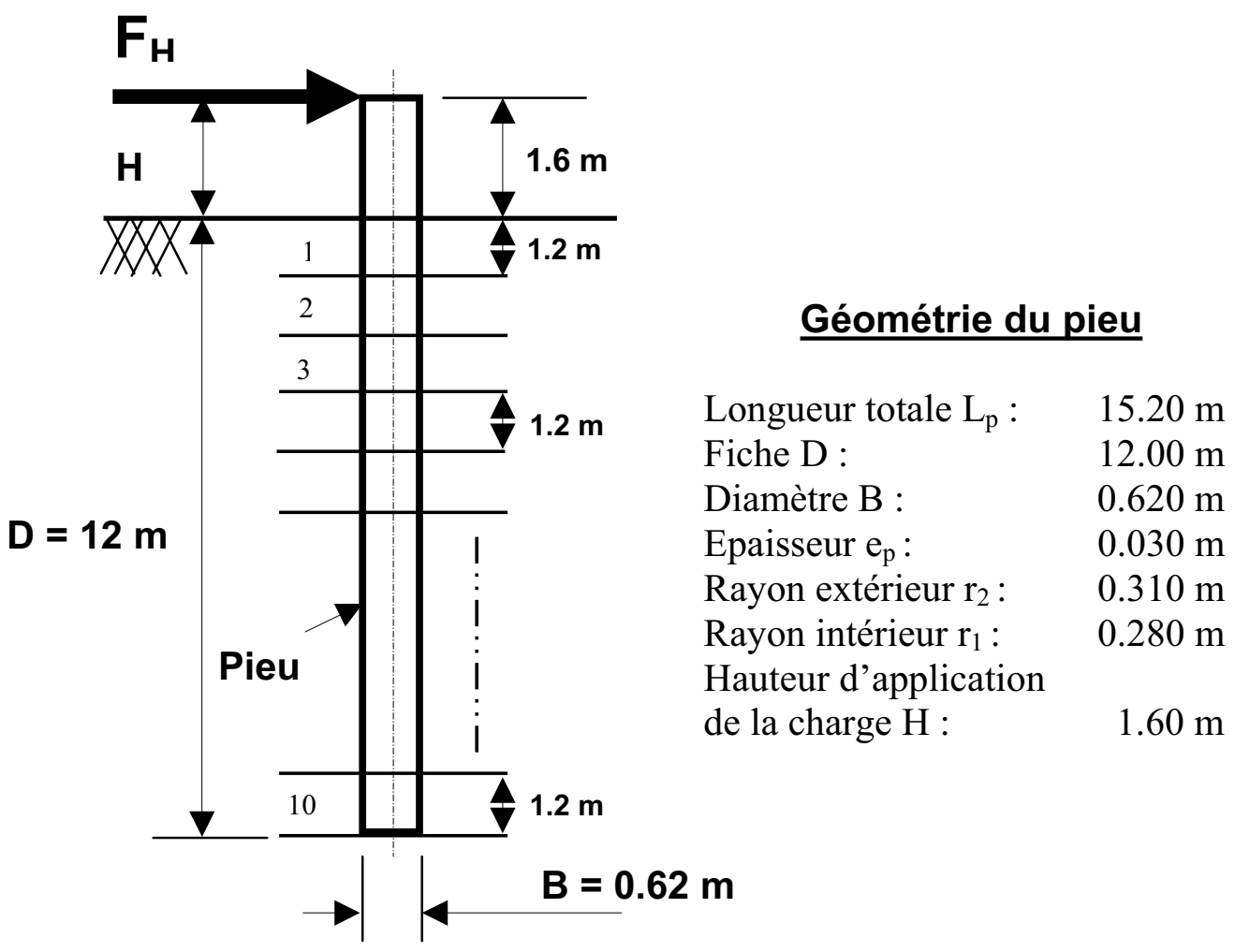

Figure 1 : Schématique du pieu prototype

Le pieu réel modélisé correspond au pieu prototype des essais effectués par Mezazigh (1995). C'est un pieu fiché sur $12 \mathrm{~m}$, de diamètre $0.62 \mathrm{~m}$, et de rigidité 
$\mathrm{E}_{\mathrm{p}} \mathrm{I}_{\mathrm{p}}$ égale à 473.6 $\mathrm{MNm}^{2}$. Le calcul numérique a été développé à l'aide du logiciel ANSYS. Le pieu et le massif de sable ont été discrétisés en 10 sous-couches d'épaisseur $1.20 \mathrm{~m}$. Sous la pointe du pieu, le sable est divisé en 2 sous-couches de même épaisseur. La charge latérale est appliquée à une hauteur de $1.60 \mathrm{~m}$ comme indiqué à la figure 1 . Les principales caractéristiques mécaniques du pieu sont rassemblées dans le tableau 1, celles correspondant au massif de sable sont données dans le tableau 2.

Tableau 1 : Caractéristiques mécaniques du pieu

\begin{tabular}{|c|c|c|c|c|}
\hline $\begin{array}{c}\text { Module d'élasticité } \\
\text { [MPa] }\end{array}$ & $\begin{array}{c}\text { Masse volumique } \\
{\left[\mathrm{kg} / \mathrm{m}^{3}\right]}\end{array}$ & $\begin{array}{l}\text { Coefficient } \\
\text { de Poisson }\end{array}$ & $\begin{array}{l}\text { Rigidité } \\
{\left[\mathbf{M N m}^{2}\right]}\end{array}$ & $\begin{array}{c}\text { Moment } \\
\text { d'inertie }\left[\mathrm{m}^{4}\right]\end{array}$ \\
\hline$E_{p}=2.110^{5}$ & $\rho_{P}=7800$ & $v_{P}=0.3$ & $\mathrm{E}_{\mathrm{p}} \mathrm{I}_{\mathrm{p}}=473.6$ & $\mathrm{I}_{\mathrm{p}}=2.2610^{-3}$ \\
\hline
\end{tabular}

Elles correspondent au sable de Fontainebleau, (Mezazigh 1995, Remaud 1999). Pour ce sable, on adopte une loi de comportement non linéaire inélastique de type Drücker-Prager. Les paramètres associés à cette loi sont au nombre de cinq, deux pour la partie élastique (module de réaction du sable $E_{S}$ et coefficient de Poisson $v_{\mathrm{s}}$ ) et trois pour la partie plastique (la cohésion C, l'angle de frottement interne $\varphi$ et l'angle de dilatance $\psi$ ). Le choix du module de réaction $E_{S}(z)$ qui évolue avec la profondeur $\mathrm{z}$ a une importance primordiale, du fait de l'influence qu'il exerce sur les courbes réponses du système pieu-sol.

Tableau 2 : Caractéristiques mécaniques du massif de sable

\begin{tabular}{|c|c|c|c|c|}
\hline $\begin{array}{c}\text { Poids volumique } \\
{\left[\mathbf{k N m}^{-3}\right]}\end{array}$ & $\begin{array}{c}\text { Coefficient de } \\
\text { Poisson }\end{array}$ & $\begin{array}{c}\text { Cohésion } \\
{[\mathbf{k P a}]}\end{array}$ & $\begin{array}{c}\text { Angle de } \\
\text { frottement }\end{array}$ & $\begin{array}{c}\text { Angle de } \\
\text { dilatance }\end{array}$ \\
\hline$\gamma_{\mathrm{d}}=16.1$ & $v_{\mathrm{s}}=0.25$ & $\mathrm{C}=16.8$ & $\varphi=39.7^{\circ}$ & $\psi=39.7^{\circ}$ \\
\hline
\end{tabular}

\section{Modélisation numérique sur sable horizontal $(\beta=0)$}

\section{1. Réalisation du maillage}

Le code ANSYS, code de calcul aux éléments finis, est particulièrement bien adapté pour la résolution des problèmes de mécanique des structures notamment grâce aux nombreuses lois de comportement implantées. Un premier maillage a été tout d'abord élaboré pour un massif de sable horizontal. Le maillage ainsi réalisé sert par la suite aux études du pieu implanté à proximité d'un talus. La structure d'ensemble est un demi-massif de sol constitué de plusieurs souscouches de sable et un demi-pieu cylindrique creux en acier.

\section{2. Lois de comportement du pieu et du sable}

Le pieu est modélisé par des éléments poutres 3D comme le recommande Mestat (1997). Ce choix s'explique par la nécessité de prendre en compte les déformations dues aux chargements latéraux du pieu. Le comportement du pieu est modélisé par une loi élastique linéaire. La modélisation par des éléments poutres nécessite de déterminer une épaisseur $\mathrm{e}_{\mathrm{p}}$, une rigidité $\mathrm{E}_{\mathrm{P}} \mathrm{I}_{\mathrm{P}}$ et un moment 
d'inertie $I_{P}$ du pieu, données imposées par le pieu modèle testé lors des études expérimentales. Le sable est modélisé par une loi élasto-plastique associée du type Drücker-Prager. Les paramètres retenus, sont ceux issus de la modélisation expérimentale en centrifugeuse, à l'exception du paramètre élastique $E_{S}$. Le coefficient de Poisson est de 0.25 , le choix de ce coefficient se justifie en se basant sur les études effectuées par Hajialilue Bonab (2003). Toutes les souscouches de sable ont un poids volumique identique, il est égal à $16.1 \mathrm{kN} / \mathrm{m}^{3}$ sur toute la profondeur z. Le modèle associé de Drücker-Prager impose $\psi=\varphi$. Dans le tableau 2 on retrouve les valeurs de ces paramètres.

\section{3. Module de réaction constant suivant la profondeur}

Plusieurs tentatives pour le calcul du module de réaction sur sable horizontal ont été effectuées pendant la modélisation numérique, dans le but d'obtenir une valeur du module de réaction qui corresponde aux déplacements expérimentaux observés. En effet, pour chaque valeur du module de réaction choisie arbitrairement, un calcul a été effectué. Les déplacements numériques obtenus en surface pour chaque cas sont comparés aux déplacements expérimentaux et ainsi de suite, jusqu'à ce qu'une valeur donne la meilleure approximation en déplacement par rapport à l'expérimental. Finalement, trois cas ont été étudiés avec un module de réaction constant suivant la profondeur $\mathrm{z}$, à savoir : $12.5 \mathrm{MPa}$, $15 \mathrm{MPa}$ et $17.5 \mathrm{MPa}$. Pour le module de réaction du sable $\mathrm{E}_{\mathrm{S}}=15 \mathrm{MPa}$, les déplacements numériques obtenus, (voir figure 2), sont presque identiques aux valeurs expérimentales. Pour les charges élevées, les écarts relatifs sont très faibles de l'ordre de $1 \%$. Les écarts relatifs sont plus significatifs pour des valeurs d'effort de moins de $500 \mathrm{kN}$.

Pour le module de réaction de $12.5 \mathrm{MPa}$, une amélioration en déplacement a été constatée pour les charges moins élevées par rapport aux résultats obtenus avec la prise en compte d'une raideur de $15 \mathrm{MPa}$. Quant aux charges plus élevées, les déplacements issus de la simulation numérique deviennent plus importants et dépassent largement les valeurs expérimentales de près de $11 \%$. Le calcul avec le module de $12.5 \mathrm{MPa}$ sur-estime les valeurs des déplacements calculés pour les charges élevées et améliore ceux des charges moins élevées. La figure 2, illustre ce constat par les deux courbes obtenus. Finalement on suppose le sable plus raide, le module de réaction est pris égal à $17.5 \mathrm{MPa}$. Pour ce cas, les déplacements numériques comparés aux déplacements expérimentaux en surface, donnent pour les charges les plus élevées des écarts relatifs de l'ordre de $7 \%$ et pour les charges les moins élevées des écarts relatifs d'environ $50 \%$. Avec ce module de réaction du sable, les déplacements numériques sont sous-estimés pour toutes les charges latérales statiques appliquées. 

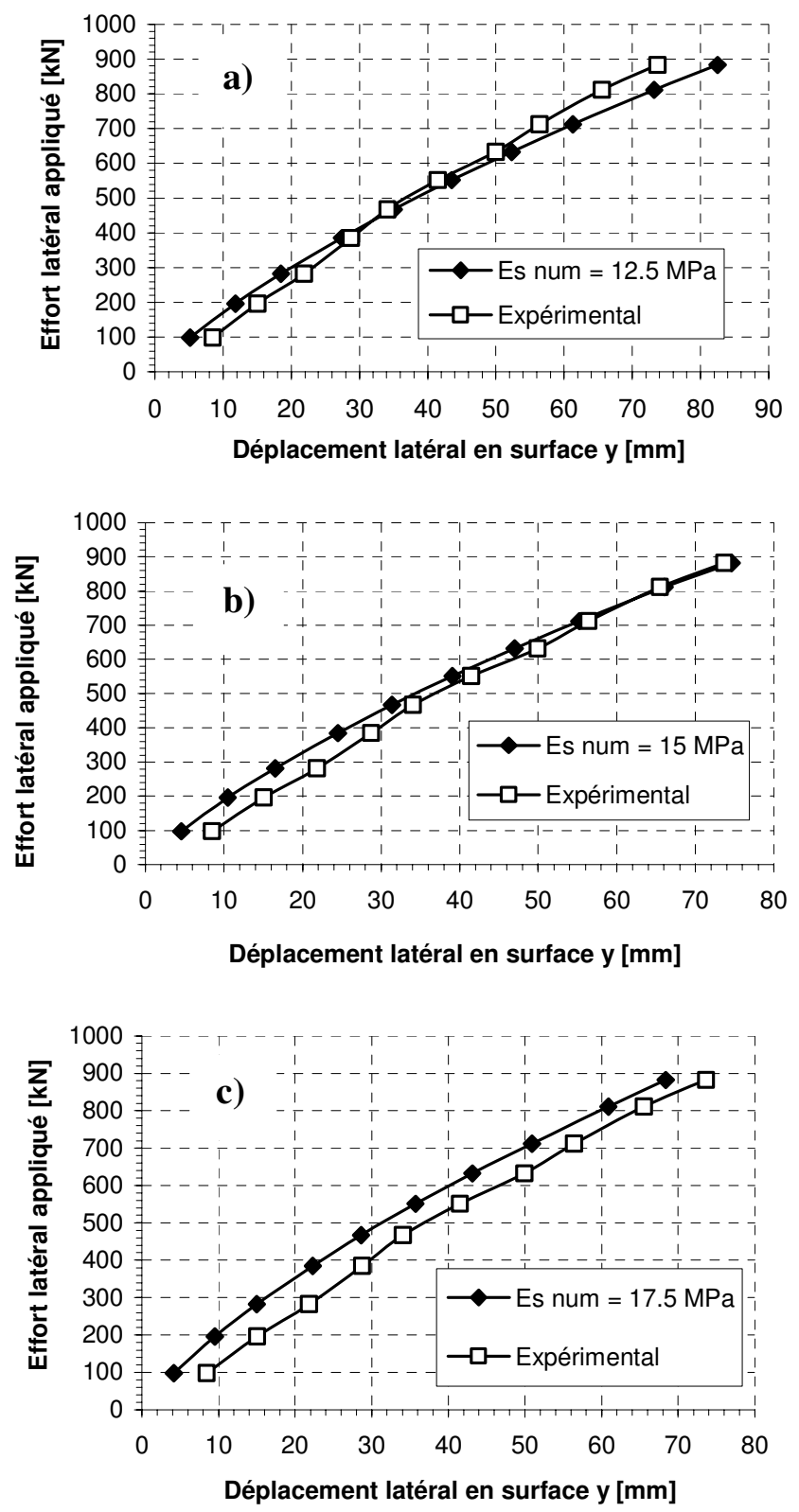

Figure 2 : Comparaison des courbes de déplacement en surface a) $\mathrm{Es}=12.5 \mathrm{MPa}, \mathrm{b}) \mathrm{Es}=15 \mathrm{MPa}, \mathrm{c}) \mathrm{Es}=17.5 \mathrm{MP}$

\section{4. Module de réaction variable suivant la profondeur}

Une nouvelle considération est proposée en prenant un module de réaction du sable variable avec la profondeur. Ce module de réaction utilise la relation non linéaire de Christoulas (1990),

$$
\mathrm{E}_{\mathrm{S}}(\mathrm{z})=0.30 \mathrm{n}_{\mathrm{H}} \mathrm{D}^{0.75} \mathrm{z}^{0.25}
$$


où $\mathrm{E}_{\mathrm{S}}(\mathrm{z})$, est le module de réaction du sable $\left[\mathrm{kN} / \mathrm{m}^{2}\right]$, D la fiche du pieu $[\mathrm{m}], \mathrm{z}$ la profondeur $[\mathrm{m}] . \mathrm{n}_{\mathrm{H}}$ définit l'état de compacité du sable $\left[\mathrm{kN} / \mathrm{m}^{3}\right]$. Pour les sols denses, le règlement Canadien (CGS, 1985) recommande d'utiliser des valeurs du coefficient $\mathrm{n}_{\mathrm{H}}$ égales à $18000 \mathrm{kN} / \mathrm{m}^{3}$, lorsqu'on se trouve au-dessus d'une nappe phréatique. En utilisant la relation [1] telle qu'elle est définie, le module de réaction varie suivant la profondeur, mais le sable devient de plus en plus raide comparativement aux modules de réaction constants. Si l'on effectue une première simulation numérique avec cette variation de $E_{S}(z)$, les déplacements numériques obtenus sont très faibles en comparaison avec les valeurs des déplacements en surface expérimentaux. Il est nécessaire de modifier la relation de Christoulas pour observer des déplacements numériques, proches des valeurs expérimentales. Pour ce faire, le massif de sable étant constitué de plusieurs souscouches de sable, le module de réaction $E_{S}(z)$ est modifié de façon à conserver le profil de variation non linéaire suivant la profondeur z. Finalement, la valeur du coefficient de 0.30 de Christoulas (1990) est modifiée. Elle devient égale à 0.11 dans la relation [1], afin de corréler au mieux les résultats numériques et expérimentaux. La nouvelle expression modifiée de Christoulas (1990) s'écrit comme suit :

$$
\mathrm{E}_{\mathrm{S}}(\mathrm{z})=0.11 \mathrm{n}_{\mathrm{H}} \mathrm{D}^{0.75} \mathrm{z}^{0.25}
$$

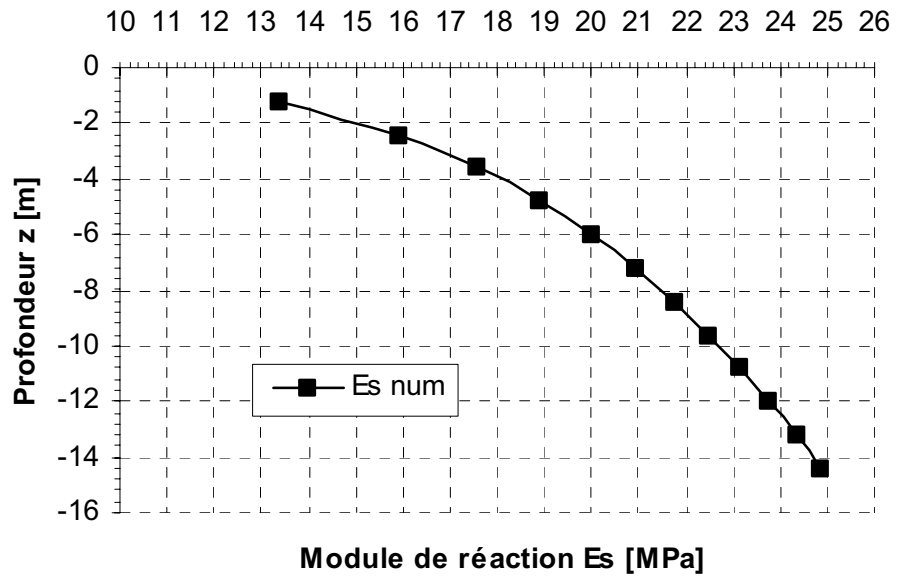

Figure 3 : Profil de variation du module de réaction $\mathrm{E}_{\mathrm{S}}(\mathrm{z})$ modifié

Cette relation a été utilisée par la suite, pour la modélisation de la partie élastique avec le modèle élasto-plastique associé de Drücker-Prager. Le profil de variation du module de réaction conforme à l'équation [2], est donné à la figure 3. Ainsi, avec le module de réaction modifié, la partie en fiche du pieu située à la profondeur z égale à $6 \mathrm{~B}$ soit $3.72 \mathrm{~m}$ est faiblement sollicitée et l'on peut penser que la contre butée est peu mobilisée au pied du pieu. 


\section{$\underline{4 .}$ Modélisation en présence d'un talus $\left(\beta_{i} \underline{\neq 0}\right)$}

\section{1. Position du problème}

Quatre pentes $\left(\beta_{\mathrm{i}} \neq 0\right)$ sont considérées pour l'étude du comportement d'un pieu isolé implanté à proximité d'un talus. Une fois le massif de sable horizontal modélisé, les paramètres issus de cette modélisation ont été adoptés pour l'étude de cas avec la présence d'un talus. Quatre pentes 2/1,3/2, 5/2 et 5/4 ont donc été retenues pour la simulation numérique. Les deux premières pentes, c'est-à-dire les pentes $2 / 1$ et $3 / 2$ correspondent respectivement à des angles de $26.6^{\circ}$ et $33.7^{\circ}$, elles correspondent aux valeurs de l'étude expérimentale en centrifugeuse. Deux autres pentes $5 / 2$ et $5 / 4$ qui correspondent respectivement à des angles de $21.8^{\circ}$ et $38.7^{\circ}$, ont été choisies pour compléter l'étude numérique.

Tableau 3 : Différents talus, pentes et distances relatives à la crête du talus

\begin{tabular}{|c|c|c|c|c|c|c|c|c|c|c|c|}
\hline $\begin{array}{c}\text { Talus } \\
\mathbf{N}^{\circ}\end{array}$ & $\begin{array}{l}\text { Pente } \\
\text { Cotan }\end{array}$ & & & & $\operatorname{tanc}$ & rel & tive & & & $\begin{array}{c}\text { Nombre } \\
\text { d'incréments }\end{array}$ & $\begin{array}{c}\text { Poids } \\
\text { volumique }\end{array}$ \\
\hline A & $2 / 1$ & $1 \mathrm{~B}$ & $2 B$ & $4 \mathrm{~B}$ & $6 \mathrm{~B}$ & $8 \mathrm{~B}$ & - & - & - & 5 part $t / B$ & 16.1 \\
\hline B & $3 / 2$ & 1B & $2 \mathrm{~B}$ & - & $6 \mathrm{~B}$ & $8 \mathrm{~B}$ & 10B & 12B & - & 5 par t/B & 16.1 \\
\hline $\mathrm{C}$ & $5 / 2$ & $1 \mathrm{~B}$ & $2 \mathrm{~B}$ & $4 \mathrm{~B}$ & $6 \mathrm{~B}$ & - & - & - & - & 5 part $t / B$ & 16.1 \\
\hline D & $5 / 4$ & - & $2 \mathrm{~B}$ & 4B & $6 \mathrm{~B}$ & $8 \mathrm{~B}$ & $10 \mathrm{~B}$ & $12 \mathrm{~B}$ & 14B & 5 part/B & 16.1 \\
\hline
\end{tabular}

Les différentes distances relatives et géométries des talus sont résumées dans le tableau 3. Pour les quatre pentes étudiées et référencées A, B, C et D les mêmes paramètres mécaniques que pour le massif de sable horizontal ont été conservés, voir tableau 2. Le module de réaction du sable est défini par la relation modifiée de Christoulas (1990) donnée par l'équation [2] et adaptée par l'introduction des coefficients de réduction $R_{P}$ relatif à la présence de talus, présenté dans le paragraphe suivant.

\section{2. Réduction du module de réaction pour les talus}

Le module de réaction pour la modélisation d'un pieu implanté à proximité d'un talus doit être réduit (Massamba 2006). La modification est basée par application des coefficients réducteur $R_{P}$ proposé par Mezazigh et Levacher (2002). Ce coefficient de réduction est une expression définie en fonction de la profondeur $\mathrm{z}$, de l'angle de talus $\beta$, de la distance à la crête du talus t et de la distance limite $\mathrm{d}_{1}$. La distance limite $d_{l}$ est donnée en fonction du diamètre $B$ du pieu. Deux expressions du coefficient de réduction $\left.\mathrm{R}_{\mathrm{P}}\right|_{2 / 1}$ et $\left.\mathrm{R}_{\mathrm{P}}\right|_{3 / 2}$ sont proposées pour définir correctement le module de réaction à utiliser en adéquation avec les pentes, à savoir :

Pour le talus A de pente 2/1, on a : 


$$
\begin{array}{r}
\left.\mathrm{R}_{\mathrm{P}}\right|_{2 / 1}=\frac{1}{2}(1-\operatorname{tg} \beta)\left[1+\frac{3 \mathrm{~d}}{\mathrm{~d}_{1}} \frac{\operatorname{tg} \beta}{1-\operatorname{tg} \beta}\right] \\
\mathrm{d}=\mathrm{t}+\frac{\mathrm{z}}{\operatorname{tg} \beta} \quad \text { et } \mathrm{d}_{1}=8 \mathrm{~B}
\end{array}
$$

avec

Pour le talus B de pente $3 / 2$, on considère:

$$
\left.\mathrm{R}_{\mathrm{P}}\right|_{3 / 2}=0.85\left(\frac{1}{2}(1-\operatorname{tg} \beta)\left[1+\frac{3 \mathrm{~d}}{\mathrm{~d}_{1}} \frac{\operatorname{tg} \beta}{1-\operatorname{tg} \beta}\right]\right)
$$

avec

$$
\mathrm{d}=\mathrm{t}+\frac{\mathrm{z}}{\operatorname{tg} \beta} \quad \text { et } \quad \mathrm{d}_{1}=12 \mathrm{~B}
$$

La distance limite $d_{1}$ est la distance pour laquelle le talus n'a plus d'influence sur le déplacement du pieu, elle est égale à $8 \mathrm{~B}$ pour un talus $\mathrm{A}$ de pente $2 / 1$ et à $12 \mathrm{~B}$ pour un talus $B$ de pente $3 / 2$. Afin de tenir compte de la réduction du module de réaction $\mathrm{E}_{\mathrm{S}}(\mathrm{z})$ du massif de sable en présence du talus, la relation modifiée de Christoulas (1990) utilisée pour le massif de sable horizontal sera multipliée pour chaque pente et pour chaque distance relative par les expressions [3] et [4]. Le coefficient de réduction $\left.R_{P}\right|_{2 / 1}$ défini pour le talus $A$ est aussi utilisé pour la modélisation du talus $C$, en considérant une distance limite comprise entre $3 \mathrm{~B}$ et 5B. Ce coefficient reste valable pour les pentes inférieures à $30^{\circ}$. Pour le talus $\mathrm{D}$, on utilise le coefficient $\left.R_{P}\right|_{3 / 2}$ qui reste aussi valable pour les pentes supérieures à $30^{\circ}$, mais en considérant cette fois une distance limite de 15B. Ainsi, pour les talus $\mathrm{C}$ et $\mathrm{D}$, on retient les mêmes coefficients de réduction que précédemment, avec des distances limites qui évoluent en fonction de la pente.

Alors, les expressions des modules de réaction réduits nécessaires à la modélisation du talus $\mathrm{A}$ de pente $2 / 1$ et $\mathrm{B}$ de pente $3 / 2$ s'écrivent sous la forme modifiée suivante :

$$
\mathrm{E}_{\mathrm{S}}(\mathrm{z})=0.11 \mathrm{n}_{\mathrm{H}} \mathrm{D}^{0.75} \mathrm{z}^{0.25} \mathrm{R}_{\mathrm{P}}
$$

Les valeurs du coefficient de réduction augmente dans la zone située en surface, jusqu'à atteindre la valeur de 1 pour une distance relative de $8 \mathrm{~B}$ pour le talus $2 / 1$ et $12 \mathrm{~B}$, pour le talus $3 / 2$. L'évolution des modules de réaction $\mathrm{E}_{\mathrm{S}}(\mathrm{z})$ pour un sable horizontal, et pour les talus $2 / 1$ et $3 / 2$, est présentée à la figure 5 illustrant ainsi la réduction qu'ils subissent lorsqu'on se trouve en présence d'un talus par rapport au massif de sable horizontal. Pour le talus $2 / 1$, il est possible de réduire le module de réaction du sable jusqu'à la profondeur z égale à $2.48 \mathrm{~m}$ soit environ 4B. Pour le talus $3 / 2$, la réduction se fait jusqu'à la profondeur $\mathrm{z}$ égale à $4.20 \mathrm{~m}$ soit environ 6.78B. Dépassé ces profondeurs, le comportement du sable devient identique à celui du module de réaction du massif de sable horizontal. 


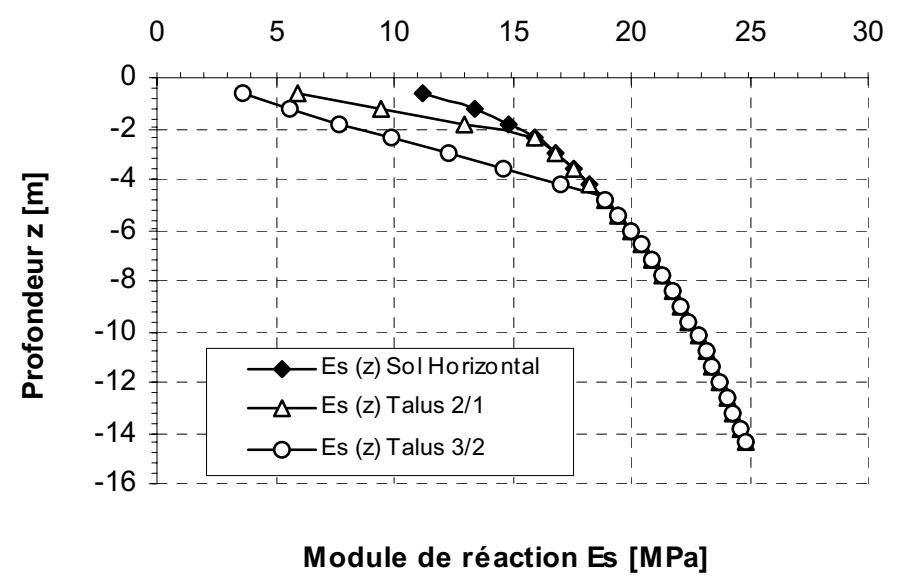

Figure 5 : Evolution des modules de réaction $E_{S}(z)$ d'un pieu isolé avec ou sans présence de talus

\section{3. Etude comparative des réponses en déplacements et des moments fléchissants}

Afin de valider la relation de Christoulas modifiée. On compare les déplacements $\mathrm{y}(\mathrm{z})$ et les moments fléchissants $\mathrm{M}_{\mathrm{f}}(\mathrm{z})$ numériques (voir figure 6) avec ceux obtenus expérimentalement par Mezazigh (1995). La prise en compte du module de réaction réduit, les déplacements numériques deviennent sensiblement égaux à ceux déduits expérimentalement, avec des écarts relatifs faibles de l'ordre de $8 \%$ pour les talus $2 / 1$ et $3 / 2$ considérés. Au vu des écarts observés, on constate bien que le module de réaction modifié simule correctement le comportement du pieu isolé implanté à proximité d'un talus.
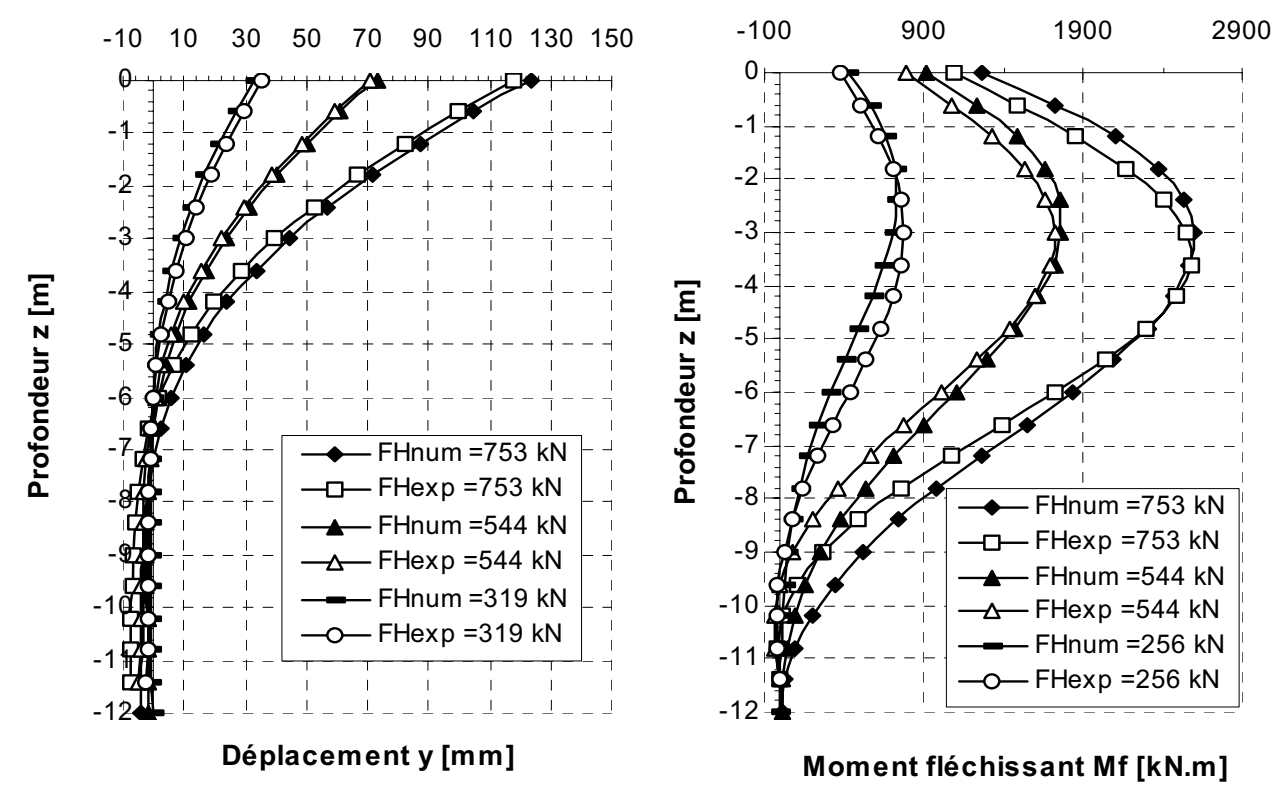

Figure 6 : Comparaison des déplacements et des moments fléchissants 


\section{Conclusions}

Les modules de réaction réduits à partir des coefficients réducteur et utilisant la relation de Christoulas modifiée, semblent très bien adaptés pour la résolution du problème numérique des pieux sous charge latérale. Les résultats obtenus sont très bons et prometteurs. Cependant, la forme géométrique du massif de sable avec pente, impose une réduction du module de réaction du sable afin que les déplacements restent assez proches de l'expérimental. Une étude particulière du module de réaction du sable avec pente à été effectuée en multipliant la relation de Christoulas modifiée par des coefficients réducteurs $\left.\mathrm{R}_{\mathrm{P}}\right|_{2 / 1}$ et $\left.\mathrm{R}_{\mathrm{P}}\right|_{3 / 2}$. Les résultats obtenus avec le module de réaction réduit pour cause de présence d'un talus utilisant la relation de Christoulas modifiée simule correctement le comportement d'un pieu. Il faut noter que dans cet article, nous nous sommes intéressés seulement aux cas des sables.

\section{$\underline{\text { Références }}$}

1 CGS Canadian Geotechnical Society (1985) "Canadian Foundation Engineering manual”. $2^{\text {nd }}$ Edition. Vancouver, pp 367-373.

2 Christoulas S. (1990) "Déplacements de pieux sous charge horizontale". Bulletin de Liaison des Laboratoire des Ponts et Chaussées. Laboratoire Centrale de Pont et Chaussées. $\mathrm{N}^{\circ}$ 168, Juillet-Août 1990. Référence 3519, pp 29-37.

2 Hajialilue Bonab M. (2003) "Modélisation physique et numérique d'un pieu isolé dans du sable soumis a un impact latéral en tête". Thèse de doctorat de l'Université de Caen. 228p.

3 Massamba F. (2006) "Modélisation numérique tridimensionnelle du comportement d'un pieu isolé chargé latéralement à proximité d'un talus". Thèse de doctorat de l'Université de Caen. 285p.

4 Mestat P. (1997) "Maillage d'éléments finis pour les ouvrages de géotechnique conseils et recommandation". Bulletin des Laboratoires des Ponts et Chaussées. 212 Nov-Déc. 1997 Référence 4161, pp 39-64.

5 Mezazigh S. et Levacher D. (2002) "Etude d'un pieu chargé latéralement a proximité d'un talus dans un massif sableux - Proposition de coefficient de réaction du sable". Apparaître dans les prochain jours.

6 Mezazigh S. (1995) "Etude expérimentale de pieux chargés latéralement: proximité d'un talus et effet de groupe". Thèse de doctorat, Université de Nantes. Ecole Centrale de Nantes, $217 \mathrm{p}$.

7 Remaud D. (1999) "Pieux sous charges latérales : étude expérimentale de l'effet de Groupe". Thèse de doctorat de l'Université de Nantes, 128p. 